\title{
Comparison of Unsteady Aerodynamic Modelling Methodologies with respect to Flight Loads Analysis
}

\author{
Thiemo M. Kier* \\ DLR German Aerospace Center, Institute of Robotics and Mechatronics, \\ 82234 Wessling, Germany
}

This paper addresses the development of aircraft models for flight loads analysis in the pre-design stage. The underlying model structure consists of the nonlinear equations of motion of a free flying, flexible aircraft, as well as a model, which calculates the distributed aerodynamics over the entire airframe.

Different possibilities in modelling the unsteady aerodynamic interactions for pre-design purposes are explored and the effects on the loads are compared in order to assess the tradeoffs between accuracy and speed. The following methods are modelled and compared:

- a quasi-steady Vortex Lattice Method (VLM) without any further unsteady improvements,

- an extended strip theory, where unsteady effects are modelled by indicial functions (IFM) such as Wagner's and Küssner's function,

- and a Rational Function Approximation according to Roger's Method of the unsteady Doublet Lattice Method (DLM).

For the comparison of the loads, the aircraft is subjected to a longitudinal gust of the shape 1-cos, in order to excite the flexible structure and unsteady aerodynamic effects. The presented comparison can serve as basis to estimate the level of uncertainty resulting from the different modelling approaches, and to choose a method according to the desired accuracy.

\section{Nomenclature}

\begin{tabular}{ll} 
Symbols & \\
$\bar{c}$ & reference chord length \\
$\bar{q}$ & dynamic pressure \\
$\mathbf{A}_{i}$ & Coefficient Matrices of RFA \\
$\mathbf{A}_{j j}$ & induced downwash matrix \\
$\mathbf{D}_{j k}^{1}$ & Differentiation Matrix for Deformations \\
$\mathbf{D}_{j k}^{2}$ & Differentiation Matrix for Deformation Rates \\
$\mathbf{D}_{f f}$ & generalized damping matrix \\
$\mathbf{I}_{b}$ & inertia tensor of aircraft \\
$\mathbf{K}_{f f}$ & generalized stiffness matrix \\
$\mathbf{K}_{g f}$ & half generalized stiffness matrix \\
$\mathbf{M}_{f f}$ & generalized mass matrix \\
$\mathbf{M}_{g h}$ & half generalized mass matrix \\
$\mathbf{P}_{g}$ & nodal forces \\
$\mathbf{P}_{g}^{a e r o}$ & aerodynamic forces \\
$\mathbf{P}_{g}^{e x t}$ & external forces \\
$\mathbf{P}_{g}^{p r o p}$ & propulsion forces \\
$\mathbf{p}_{j}$ & aerodynamic preassure acting on panel \\
$\mathbf{Q}_{j j}$ & AIC matrix \\
$\mathbf{S}_{k j}$ & Aerodynamic Integration Matrix \\
$\mathbf{T}_{b E}$ & transformation inertial to body fixed \\
$\mathbf{u}_{f}$ & generalized coordinates \\
$\mathbf{V}_{b}$ & velocity vector \\
\hline
\end{tabular}

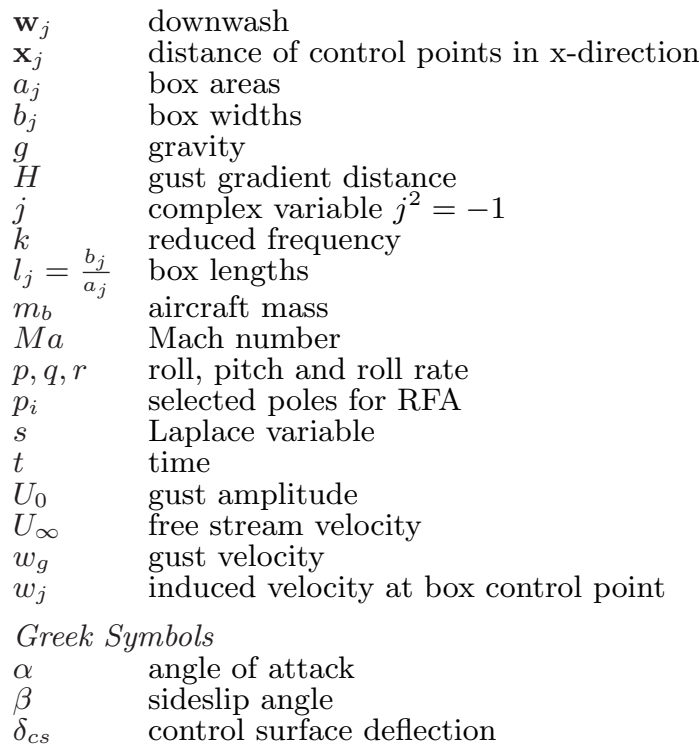

*Research Engineer, AIAA Member, Thiemo.Kier@dlr.de 


$\begin{array}{ll}\gamma_{j} & \text { dihedral angle (relative to gust) } \\ \boldsymbol{\Gamma}_{j} & \text { circulation of boxes } \\ \boldsymbol{\Omega}_{b} & \text { angular velocity vector } \\ \mathbf{\Phi}_{g b} & \text { modal matrix for rigid body modes } \\ \boldsymbol{\Phi}_{g f} & \text { modal matrix for flexible modes } \\ \omega & \text { circular frequency } \\ \omega & \text { circular frequency } \\ \Phi(\tau) & \text { Wagner function approx. } \\ \phi, \theta, \psi & \text { Euler angles } \\ \Psi(\tau) & \text { Küssner function approx. } \\ \rho & \text { air density } \\ \tau & \text { reduced time } \\ \text { Abbreviations } \\ \text { AIC } & \text { Aerodynamic Influence Coefficient Matrix } \\ \text { CG } & \text { Center of Gravity } \\ \text { DLM } & \text { Doublet Lattice Method } \\ \text { DOF } & \text { Degree(s) of Freedom } \\ \text { EoM } & \text { Equations of Motion } \\ & \end{array}$

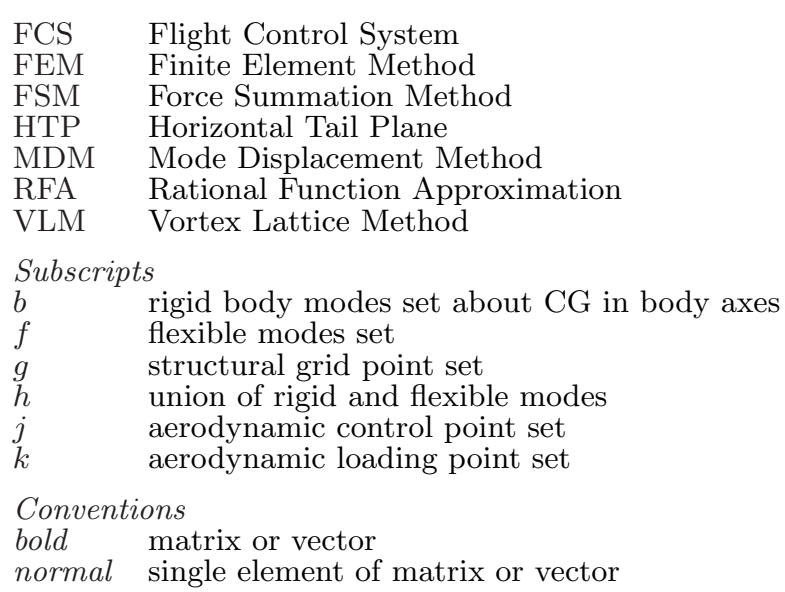

\section{Introduction}

For certification of an aircraft, it has to be demonstrated that its structure can withstand the loads acting on it without damage. In order to design the structure accordingly, a so called loads envelope has to be computed. This loads envelope is comprised of critical combinations of flight conditions, (e.g. altitude, Mach number), mass configurations and excitations (e.g. manoeuvres, gusts, dynamic landing). These so called load cases are specified for commercial aircraft in the FAR/JAR Part 25 Regulations. ${ }^{1}$

The inputs for loads analysis models are generally a Finite Element Model (FEM) containing the stiffness and mass data for each mass case, the distributed steady and/or unsteady aerodynamic loading at the specified Mach number, control laws of the Flight Control System (FCS) influencing the control surface deflections, dependent on the current flight, and information about the systems, such as actuator transfer functions. The model is then subjected to external disturbances, such as gusts, or excited by pilot inputs.

A variable aircraft model simulation environment for special flight loads investigations, named VarLoads ${ }^{2}$ has been presented previously. The underlying model structure consists of the nonlinear equations of motion of a flexible aircraft, as well as a model that computes distributed aerodynamic forces and moments over the airframe, allowing for direct computation of aerodynamic and inertial loads at airframe locations of interest. Furthermore, engine, actuation, and atmospheric models are included. VarLoads ${ }^{2}$ is a MATLAB/SIMULINK based simulation environment for flight dynamics and loads analysis of elastic aircraft. The main emphasis of VarLoads is on flexibility and modularity, making it particularly suitable for special investigations, where short model update cycles are required. Unlike other aeroelastic simulations in pre-design, this environment is loop capable and is targeted towards calculating large amounts of load cases in the order of several hundreds rather than doing a single analysis with a chosen load case, which is believed to be the sizing case. This approach is closer to the actual design process of commercial aircraft and ensures a smooth transition from the predesign stage to the actual design load loop calculation, preventing unpleasant surprises (e.g. the assumed sizing load case turns out not to be the actual sizing load case).

The scarcity of available data during pre-design and the necessity to explore many design alternatives, require a robust and fast implementation of methods with a known uncertainty level rather than much slower high fidelity methods. Usually only quasi-steady aerodynamics are employed for this purpose. Nevertheless, consideration of unsteady aerodynamic effects is of particular importance during rapid manoeuvres and gust conditions in order to accurately predict the occurring flight loads. This paper explores different possibilities in modelling the unsteady aerodynamic interactions for pre-design purposes and compares the effects on the loads in order to assess the tradeoffs between accuracy and speed. The methods that compared are a quasi-steady Vortex Lattice Method (VLM), an extended strip theory, where unsteady effects are modelled by indicial functions (IFM) such as Wagner's and Küssner's function, and, as reference, a Rational Function Approximation according to Roger's Method of the unsteady Doublet Lattice Method (DLM).

In the following sections the flight loads analysis models will be developed. The used Equations of Motion (EoM) and the corresponding load equation will be introduced. The necessary inputs for the EoM stem from a structural FEM model. Then the aerodynamic theories are introduced and it is explained how the loads are transferred to the structural gird. After trim calculations the simulations are performed for all methods 
and the loads are compared.

\section{Equations of Motion}

One of the main motivations for the development of the simulation environment VarLoads was the desire to have one common model, that is capable of handling the large nonlinear motions of a manoeuvre, as well as the small perturbations resulting from the flexibility of the structure. Therefore, equations of motion based using so called "mean axes" as body reference system ${ }^{3,4}$ were implemented in VarLoads.

$$
\begin{aligned}
{\left[\begin{array}{c}
m_{b}\left(\dot{\mathbf{V}}_{b}+\boldsymbol{\Omega}_{b} \times \mathbf{V}_{b}+\mathbf{T}_{b E}\{0,0, g\}^{T}\right) \\
\mathbf{I}_{b} \dot{\boldsymbol{\Omega}}_{b}+\boldsymbol{\Omega}_{b} \times\left(\mathbf{I}_{b} \boldsymbol{\Omega}_{b}\right)
\end{array}\right] } & =\boldsymbol{\Phi}_{g b}^{T} \mathbf{P}_{g}^{e x t} \\
\mathbf{M}_{f f} \ddot{\mathbf{u}}_{f}+\mathbf{D}_{f f} \dot{\mathbf{u}}_{f}+\mathbf{K}_{f f} \mathbf{u}_{f} & =\boldsymbol{\Phi}_{g f}^{T} \mathbf{P}_{g}^{e x t}
\end{aligned}
$$

These equations consist of the nonlinear rigid body equations of motion, as used by the flight mechanics community, and the linear elastic equations of motion used in structural dynamics. The coupling of the rigid and flexible parts is driven solely by the external forces and moments $\left(\mathbf{P}_{g}^{\text {ext }}=\mathbf{P}_{g}^{\text {aero }}+\mathbf{P}_{g}^{\text {prop }}\right)$ originating from, e.g., the aerodynamics and propulsion.

The derivation is based on the Lagrange equations with following simplifying assumptions.

1. the structure is composed of concentrated masses

2. Hooke's law is valid, i.e. deformations are small

3. Eigenvectors are available from a modal analysis and are orthogonal w.r.t. the mass matrix

4. deformations and rate of deformations are co-linear, i.e. their cross product can be neglected

5. the tensor of inertia is assumed constant

6. an earth fixed inertial reference system is assumed

7. gravity is uniform within this reference system

For a detailed derivation of the equations of motion and the underlying assumptions, please refer to the papers by Waszak and Schmidt ${ }^{3,4}$ or Reschke. ${ }^{5}$

\section{Load Recovery}

In order to recover the nodal loads acting on the condensed structural grid, the force summation method (FSM), ${ }^{6}$ also referred to as mode acceleration method, is employed. The Force Summation Method requires the external forces to be available on the structural grid, i.e. the AIC matrix has to be available in a half generalized form.

$$
\mathbf{P}_{g}=\mathbf{P}_{g}^{e x t}-\mathbf{M}_{g h}\left[\begin{array}{c}
\mathbf{T}_{b E}\{0,0, g\}^{T}+\boldsymbol{\Omega} \times \mathbf{V}_{b}+\dot{\mathbf{V}}_{b} \\
\dot{\boldsymbol{\Omega}}_{b} \\
\ddot{\mathbf{u}}_{f}
\end{array}\right]
$$

Alternatively, the mode displacement method (MDM) can be used to recover the nodal loads, where the modal displacements are multiplied with the half generalized stiffness matrix. The mode displacement method requires only a fully generalized AIC matrix, since the loads are deduced from the displacements rather than the summation of external forces, reducing the problem size considerably. However the mode displacement method has a poor convergence behavior, ${ }^{7}$ so the force summation method is preferred.

$$
\mathbf{P}_{g}=\mathbf{K}_{g f} \mathbf{u}_{f}
$$

Integrated loads are calculated by summing the outboard nodal loads at previously defined monitoring stations. The loads envelope is formed by sorting the integrated loads, yielding the sizing load cases for every grid point degree of freedom (DOF), as well as the time correlated loads. 


\section{Structural Model}

The structural model for loads analyses is usually a FEM model to which the masses (systems, payload, fuel and structural) are added separately rather than modelling the elements with density properties. This approach has been chosen to decouple the mass estimation method from the FEM model, which is required for all non-structural masses as well. The model is then statically condensed using Guyan ${ }^{8}$ reduction, yielding the condensed structural grid $g$-set. Subsequently a modal analysis is performed to obtain the eigenvalues and eigenvectors, serving as input for the equations of motion.

\section{Aerodynamics}

One of the key aspects of the loads analysis is the calculation of aerodynamic forces acting on the structure. A vast amount of theories is available, ranging from simple lifting line to high fidelity NavierStokes CFD solvers. As pointed out before, the large amount of load cases that have to be considered in a dynamic simulation are prohibitive for costly calculations. Therefore, usually classical methods derived from potential theory, such as the Vortex Lattice Method $^{9}$ (VLM) are employed. Those linear methods can then be corrected with a small number of CFD calculations at points in the flight envelope where nonlinearities are expected. These aerodynamic nonlinearities are usually found in the high Mach number region.

When gust loads are to be calculated, unsteady aerodynamics have to be considered. The standard method is the Doublet Lattice Method ${ }^{10}$ (DLM), which solves the acceleration potential equations in the frequency domain. In order to use the results in the time domain, a rational function approximation (RFA) has to be carried out, as described by Roger ${ }^{11}$ or Karpel. ${ }^{12}$ In the light of predesign applications it is also common to model the unsteadiness of the flow field with transfer functions. The so called Wagner function is used for sudden changes in angle of attack and the Küssner function ${ }^{13}$ for intrusion in sharp edged gusts. The use of these functions considerably reduces the modelling effort.

\section{A. Interpolation between Structural and Aerodynamic Grid}

In order to apply the calculated aerodynamic forces, they have to be transformed to the structural grid points. Since usually the aerodynamic and structural grids do not coincide, a methodology for the transfer of loads and displacements between the grids has to be employed. This is referred to as splining.

Mauermann ${ }^{14}$ developed a linear splining method based on beam shape functions, which is capable of handling kinks in the structural axis. The force acting points of the aerodynamic grid ( $k$-set) are projected orthogonally onto the structural axis (comprised of the structural nodes (g-set)) and are attached rigidly. When applying this methodology a so called spline matrix $\mathbf{T}_{k g}$ which linearly relates the deformations of the structural grid to that of the aerodynamic grid is computed. Energy considerations yield that loads are transferred from the aerodynamic grid to the structural grid by simply multiplying with the transpose of the spline matrix $\mathbf{T}_{k g}^{T}$.

This method is applied in the present work for all methods described in the following sections.

\section{B. Quasi-Steady Vortex Lattice Method}

The $\mathrm{VLM}^{9}$ is a method solving the velocity potential equations, resulting in a so called aerodynamic influence coefficient (AIC) matrix. The AIC matrix relates the velocity at a control point to forces or pressures at an acting point. This (quasi-)steady AIC matrix is particularly suitable for time domain simulations, since it has to be computed only once for each Mach number during the preprocessing. The varying forces during a dynamic simulation can then be calculated by multiplication with the velocity vectors at the control point that are computed from the rigid body and elastic motions. The computation of the aerodynamic loading reduces to a mere matrix multiplication.

The VLM models lifting surfaces. Therefore, volumetric bodies are idealized with a cruciform shape. An example of a Vortex Lattice grid for predesign studies is shown in Figure 1.

The VLM discretizes lifting surfaces as small elementary wings (boxes), which consist of a horseshoe shaped vortex line at the quarter chord and a control point. At this control point, which is located at the $75 \%$ chord, the normal component of the velocity has to be zero, since there is no flow through the solid surface. In order to satisfy this boundary condition, the circulation strengths of the horseshoe vortices have to be set accordingly, so that their induced velocity compensates the normal component $w$ of the velocity 


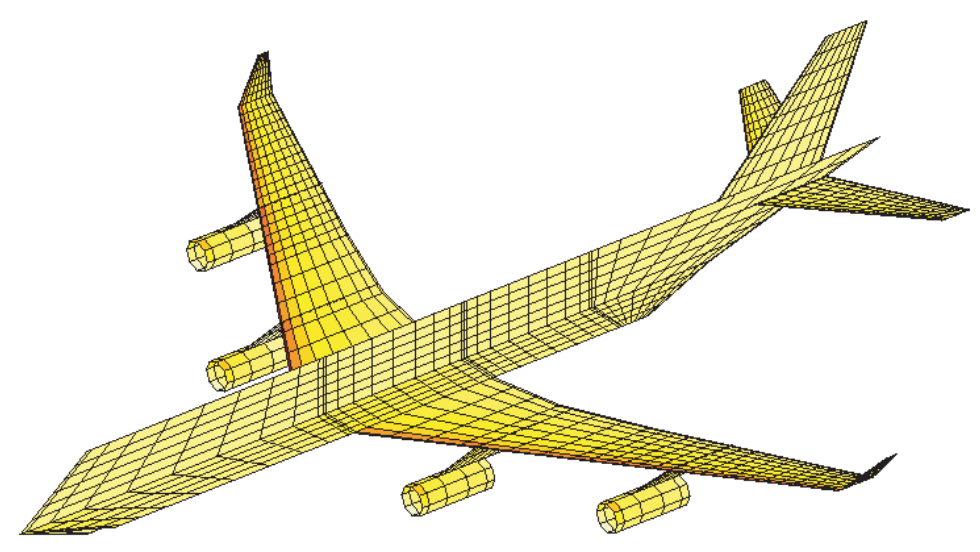

Figure 1. Vortex lattice grid for predesign studies.

resulting from rigid body and elastic motion of the aircraft. The matrix $\mathbf{A}_{j j}$ relates the circulation strengths of the horseshoe vortices to the induced velocities at the control points as computed by the Biot-Savart-Law.

$$
\mathbf{w}_{j}=\mathbf{A}_{j j} \boldsymbol{\Gamma}_{j}
$$

When the circulation strengths are known, the resulting aerodynamic pressures can be computed with the Kutta-Joukowsky theorem.

$$
\mathbf{p}_{j}=\rho U_{\infty} \boldsymbol{\Gamma}_{j} \mathbf{l}_{j}
$$

combining 5 and 6 , yields the relationship between the local angle of attack and the local pressure:

$$
\mathbf{p}_{j}=\bar{q} \mathbf{Q}_{j j}\left\{\frac{\mathbf{w}_{j}}{U_{\infty}}\right\}
$$

\section{Quasi-steady Aerodynamic Loads}

Once the pressures have been calculated they have to be applied as discrete forces and moments to be transformed to loads on the structural grid via the spline matrix $\mathbf{T}_{k g}$. The aerodynamic grid $(k$-set) is located in the box centers. It consists of a translational and rotational degree of freedom for each box and is therefore twice the size of the ( $j$-set). The matrix $\mathbf{S}_{k j}$ relates the pressures to the $k$-set loads by multiplication with the box area. The resulting force is acting on the quarter chord point of each box. In order to apply the loads at the box mid point, the force has to be multiplied with the lever arm $l_{j} / 4$ of each box.

$$
P_{k}=\underbrace{\left[\begin{array}{c}
a_{j} \\
a_{j} l_{j} / 4
\end{array}\right]}_{S_{k j}} p_{j}
$$

In the steady case the vector $\mathbf{u}_{x}$ contains the rigid body motions $\alpha, \beta, p, q$ and $r$ as well as control surface deflections $\delta_{c s}$. The matrix $\mathbf{D}_{j x}$ transforms the vector $\mathbf{u}_{x}$ to local angles of attack of each box by scalar multiplication with the normal vector of each box $n_{j}$.

The matrix $\mathbf{D}_{j k}^{1}$ relates the deformation to a local angle of attack $u_{j}$ by simply picking the rotational and omitting the translational degree of freedom of the $k$-set. 


$$
u_{j}=\underbrace{\left[\begin{array}{l}
0 \\
1
\end{array}\right]}_{D_{j k}^{1}} u_{k}
$$

The matrix $\mathbf{D}_{j k}^{2}$ relates the rate of deformation to a local angle of attack $u_{j}$. The lever arm $l_{j} / 4$ is the distance of the box mid point of the $k$-set to the $75 \%$ chord control point of the $j$-set.

$$
u_{j}=\underbrace{\left[\begin{array}{c}
1 \\
l_{j} / 4
\end{array}\right]}_{D_{j k}^{2}} \frac{\dot{u}_{k}}{U_{\infty}}
$$

The quasi steady aerodynamic loads with contributions from rigid body modes, control surface modes, flexible deformation and rate of flexible deformation can be summarized by following equation:

$$
\mathbf{P}_{g}^{a e r o}=\bar{q} \mathbf{T}_{k g}^{T} \mathbf{S}_{k j} \mathbf{Q}_{j j}(\underbrace{\mathbf{D}_{j x} \mathbf{u}_{x}}_{\text {rb and cs modes }}+\underbrace{\mathbf{D}_{j k}^{1} \mathbf{T}_{k g} \boldsymbol{\Phi}_{g f} \mathbf{u}_{f}}_{\text {deformation }}+\underbrace{\mathbf{D}_{j k}^{2} \mathbf{T}_{k g} \boldsymbol{\Phi}_{g f} \frac{\dot{\mathbf{u}}_{f}}{U_{\infty}}}_{\text {deformation rate }})
$$

\section{Quasi-Steady Gust Loads}

The gust influence is accounted for by delaying the gust by the time it takes to reach each of the control points of the $j$-set. To reduce the number of required delays, the aircraft can be divided into streamwise zones, where the gust velocity is assumed to have the same magnitude. The lifting force will act immediately with its steady value as soon as the gust arrives at the control point.

\section{Unsteady Strip Theory}

When the aircraft performs rapid maneuvers or encounters turbulence, the aerodynamic forces do not reach their steady value immediately, but rather approach that value asymptotically after a certain time. The Kelvin-Thomson theorem states that the overall circulation of a system cannot change over time. A classical example is the starting vortex: the sudden acceleration of a resting airfoil generates circulation. Since the overall circulation does not change over time, a vortex of equal strength but opposite sign is shed at the trailing edge of the airfoil. This shed vortex induces a velocity which counteracts the bound (lift generating) vortex. But since the shed vortex moves away from the airfoil with the free stream velocity, its influence on the airfoil degrades over time and the lift approaches the steady values. This behavior can be is described by the so called Wagner function. The equivalent function for gust encounters is the Küssner function, which differs from the Wagner function in that way that the relative angle of attack changes not immediately over the entire airfoil, but rather gradually as the airfoil penetrates the gust. The functions describing the time dependency of the aerodynamic lift are called indicial functions. Therefore, this methodology of modelling unsteady effects will be referred to as Indicial Function Method (IFM).

\section{Indicial Lift Functions}

The Wagner and Küssner function can be derived analytically. However, it is advantageous to approximate these functions, in order to have a convenient form available for simulations. Common exponential approximations $^{13}$ for the Wagner, respectively the Küssner function are given as:

$$
\begin{aligned}
& \Phi(\tau)=1.0-0.165 e^{-0.0455 \tau}-0.335 e^{-0.3 \tau} \\
& \Psi(\tau)=1.0-0.5 e^{-0.13 \tau}-0.5 e^{-1.0 \tau} \quad, \text { with } \tau=\frac{2 U_{\infty}}{\bar{c}} t
\end{aligned}
$$


The Wagner and Küssner functions are depicted in Figure 2. The parameter $\tau$ is the reduced time, which can also be regarded as the distance of the travelling airfoil in semi-chords. The Küssner function is applied to the steady strip loads, as soon as the leading edge penetrates the gust. The time delays are modelled according to the distance of the aircraft nose to the leading edge of the chordwise strips.

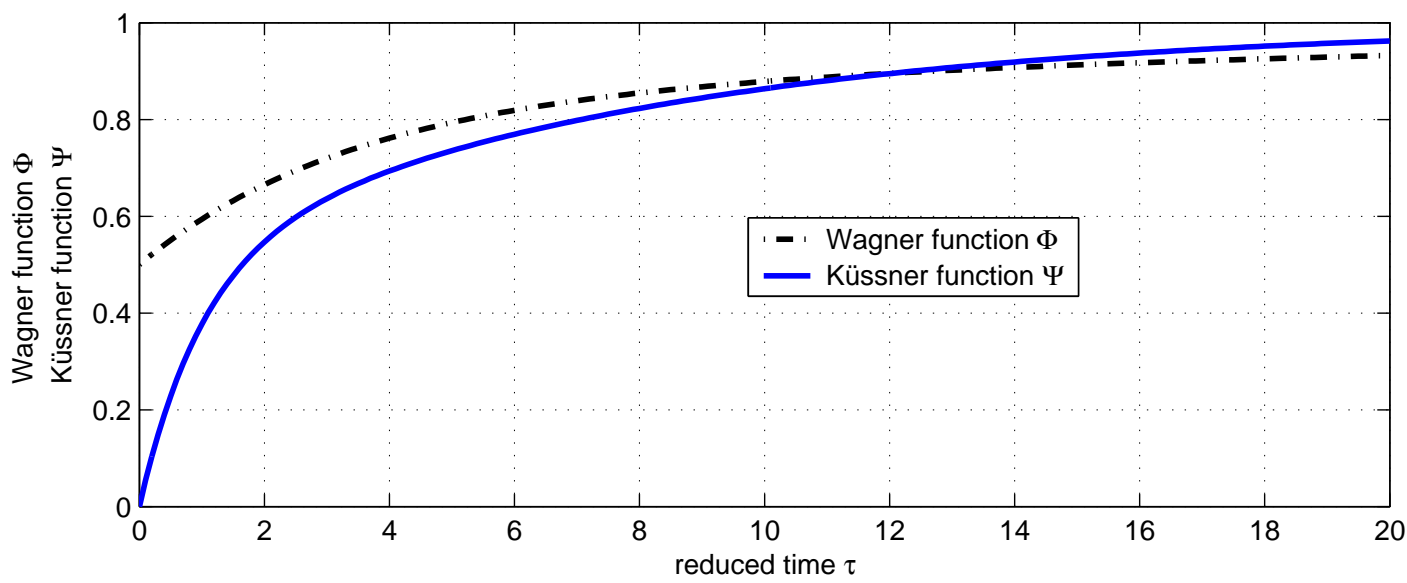

Figure 2. Wagner $\Phi(\tau)$ and Küssner $\Psi(\tau)$ function vs. reduced time $\tau$

\section{Steady Strip Loads}

Since the indicial functions are applied to entire chordwise strips, the matrices from the steady vortex lattice method have to be modified slightly. The aerodynamic load application points $(k$-set) are redefined at the quarter chord of each strip. Therefore the size of the new $k$-set reduces to two times the number of strips. Equation 8 for the integration matrix $\mathbf{S}_{k j}$ is changed accordingly. The area corresponds to the sum of all box areas that comprise a strip and the lever arms are the distances from all box quarter chord locations to the quarter chord location of the strip.

The spline matrix $T_{k g}$ now connects the quarter chord locations of each strip with the structural axis. The rotational degree of freedom of matrix $\mathbf{D}_{j k}^{1}$ of eq. 9 and the translational DOF of matrix $\mathbf{D}_{j k}^{2}$ of eq. 10 have to be summed up for the boxes comprising a strip. The lever arms for the rotational DOF of matrix $\mathbf{D}_{j k}^{2}$ are now the distances of the strip quarter chord to the control points of the contributing boxes. It has to be noted that this changes the splining considerably. Instead of connecting each box mid point orthogonally with the structural axis, only the quarter chord points of the strips are connected in that way. The movement of the control points of the strips are realized by a rigid connection with the strip $k$-set in streamwise direction. Figure 3 illustrates the aspects of the different ways of splining.

\section{Unsteady Doublet Lattice Method}

As well as the Vortex Lattice Method, the Doublet Lattice Method is based on the equations of potential theory, but additionally includes the unsteady terms. In the steady case the DLM reduces to the VLM. The discetization scheme is identical. Instead of the velocity potential equations as the VLM, the DLM solves the acceleration potential equations using a harmonic approach, resulting in a solution in the frequency domain. The resulting AIC matrices are computed at discrete reduced frequency points and are complex and fully occupied.

$$
\mathbf{Q}_{j j}(M a, k)
$$

with the reduced frequency

$$
k=\frac{\omega \bar{c}}{2 U_{\infty}}
$$

In order to make use of the resulting AIC matrices in the time domain a so called Rational Function Approximation (RFA) has to be carried out. 


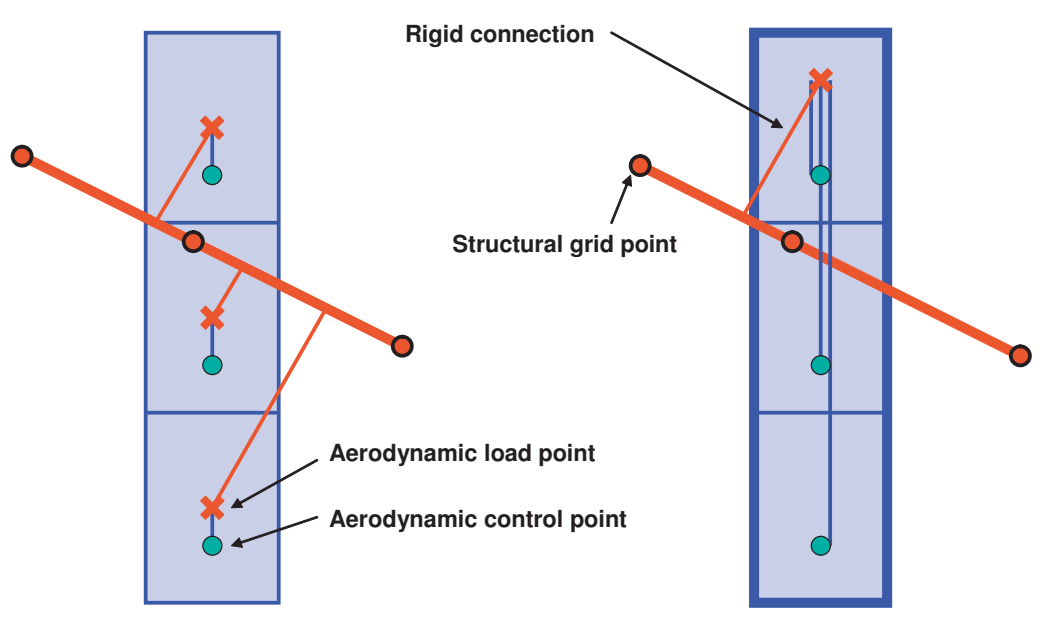

Figure 3. Splining of boxes vs. splining of strips

\section{Rational Function Approximation}

The RFA transforms complex aerodynamic loads into the time domain, by fitting rational functions with a least squares method. According to Roger, ${ }^{11}$ the rational function is assumed to be of the following form:

$$
\mathbf{Q}(k)=\mathbf{A}_{0}+\mathbf{A}_{1} j k-\mathbf{A}_{2} k^{2}+\sum_{i=1}^{n p} \mathbf{A}_{i+2} \frac{j k I}{j k+p_{i}},
$$

where $p_{i}$ are user selected poles. The terms $\mathbf{A}_{2+i}$ represent the so called aerodynamic lag states, which model the delay effects of the aircraft wake. Once the the discrete frequency points have been approximated as rational function, they can be written in terms of the Laplace variable $s$ in the following way:

$$
\mathbf{Q}(s)=\mathbf{A}_{0}+\mathbf{A}_{1} s+\mathbf{A}_{2} s^{2}+\mathbf{D}(s \mathbf{I}-\mathbf{R})^{-1} \mathbf{E} s
$$

\section{Rigid Body and Flexible Motion}

Since the DLM matrices are computed in a geodetic reference frame, the rigid body modes have to be corrected in order to decouple heave from pitch effects, respectively lateral displacement from yaw effects. ${ }^{15}$

The uncorrected rigid body modes about the CG in the body reference frame are given by:

$$
\mathbf{\Phi}_{g b}=\left[\Phi_{x}, \Phi_{y}, \Phi_{z}, \Phi_{\phi}, \Phi_{\theta}, \Phi_{\psi}\right]
$$

The corrected form subtracts the translational displacement from the rotation in order to remove the angel of attack, respectively sideslip effects.

$$
\hat{\mathbf{\Phi}}_{g b}(k)=\left[\Phi_{x}, \Phi_{y}, \Phi_{z}, \Phi_{\phi}, \Phi_{\theta}+\frac{\bar{c}}{j k} \Phi_{z}, \Phi_{\psi}-\frac{\bar{c}}{j k} \Phi_{y}\right]
$$

Focusing on the vertical movement, that means that the angle of attack is then covered by the heaving motion $\dot{z}$ and the pitch rate $q$ can be simply applied to the $\dot{\theta}$ term, which is now decoupled from the angle of attack.

Before performing the RFA for the rigid body modes the corrected frequency dependent mode shapes have to be right multiplied to arrive at the half generalized AIC matrix

$$
\mathbf{Q}_{g b}(k)=\mathbf{Q}_{g g}(k) \hat{\mathbf{\Phi}}_{g b}(k)
$$

The half generalized AIC matrix for the flexible modes simply is given by

$$
\mathbf{Q}_{g f}(k)=\mathbf{Q}_{g g}(k) \mathbf{\Phi}_{g f}
$$




\section{Control Surface Modes}

When control surface rates are considered, additionally induced velocities from the control surface motion have to be accounted for. I.e. the distances of the control points of the control surface from the hinge line have to be calculated. The local angle of attack due to control surface deflection is computed similarly to the steady case. The contributions are summarized in the control surfaces modal matrix $\boldsymbol{\Phi}_{k x}$. Note that in the unsteady case the $x$-set contains only the control surfaces, since angle of attack $\alpha$, sideslip angel $\beta$ and the rates $p, q, r$ are accounted for by the rigid body modes.

$$
\mathbf{Q}_{g x}(k)=\mathbf{T}_{k g}^{T} \mathbf{S}_{k j} \mathbf{Q}_{j j}(k)\left(\mathbf{D}_{j k}^{1} \boldsymbol{\Phi}_{k x} \mathbf{u}_{x}+\mathbf{D}_{j k}^{2} \boldsymbol{\Phi}_{k x} \frac{\dot{\mathbf{u}}_{x}}{U_{\infty}}\right)
$$

\section{Unsteady Gust Loads}

The gust velocity with the associated delays can be described in the frequency domain as follows

$$
\mathbf{w}_{j}(\omega)=\cos \left(\gamma_{j}\right) e^{-j \omega\left(\mathbf{x}_{j}\right) / U_{\infty}}
$$

However approximating the gust column as rational function is a difficult task because of the spiraling nature of the phase shifts. When the gust calculations are calculated in a nonlinear simulation environment, the delays can also be modelled directly. Similarly to the steady case, the aircraft can be divided into streamwise zones, where the gust velocity is assumed to have the same magnitude. This is of particular importance, since the number of lag states increases linearly with the number of zones. The AIC matrix that has to be approximated for the gust is given by:

$$
\mathbf{Q}_{g j}(k)=\mathbf{T}_{k g}^{T} \mathbf{S}_{k j} \mathbf{Q}_{j j}(k) \mathbf{T}_{j j_{z o n e}}
$$
in. $^{12}$

Alternatively, it is possible to approximate a gust column for each of the zones. This approach is described

\section{Trimming}

The starting conditions for a dynamic manoeuvre have to be set, so that the aircraft is in an equilibrium state. Usually this state is the horizontal flight, where the forces and moments are balanced.

$$
\begin{aligned}
\dot{x} & =f(x, u) \\
y & =g(x, u)
\end{aligned}
$$

Mathematically the nonlinear system of equations 25 has to be solved, where the states are defined by the equations of motion. The inputs are generally the control surfaces deflections and the engine thrust setting. The outputs are chosen according to the requirements for the flight condition, e.g. angle of attack $\alpha$, sideslip angle $\beta$, Mach number $M a$, and load factor $N_{z}$.

In order to compute a determined solution, the number of free variables has to equal the number of equations of the given system. When the constraints are set accordingly, a trim routine, based on the MINPACK library, ${ }^{16}$ solves the system. The trim solution defines the initial conditions for the simulation.

Additionally the inital conditions for the unsteady aerodynamics have to be determined, i.e. the steady state values are used to initialize the unsteady aerodynamics of the aircraft motion.

\section{Simulations}

For the comparison of the different modelling methodologies, the aircraft is subjected to a longitudinal gust of the shape 1-cos, according to JAR/FAR 25.341. ${ }^{1}$

The quantity observed is the wing root bending moment. The model of a generic transport category aircraft consists of a condensed structural model with approx. 750 DOF. For the simulations 40 elastic modes are selected. The AIC matrices for the VLM and the DLM have been calculated at a Mach number of zero, to avoid differences of compressibility corrections between the potential theory methods and the 
indicial functions. The velocity of the aircraft has then been set to $M a=0.3$ at sealevel $\left(V_{\text {tas }}=102 \frac{\mathrm{m}}{\mathrm{s}}\right)$. For similar reason, the fuselage is not modelled, since indicial functions are available for airfoils only. The panelling scheme used (approx. 1400 boxes corresponding to approx. 150 chordwise strips) is depicted in Figure 4. No initial downwash is given, i.e. there is no prescribed zero-lift-distribution and the zero-lift angle of attack is $0^{\circ}$.

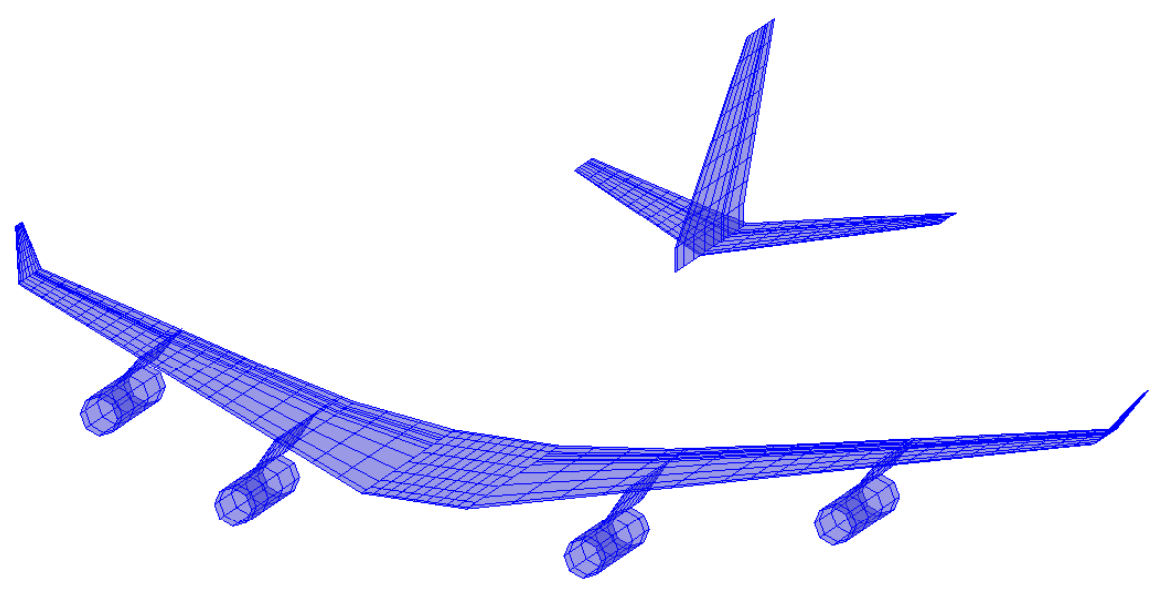

Figure 4. DLM/VLM panelling scheme

The IFM introduces 870 additional states (290 Wagner / 580 Küssner) for the unsteady aerodynamics. The RFA of the DLM introduces 3380 aerodynamic lag states (230 motion / 3150 gust). This is the maximum number for the gust lag states, where each zone contains only one distance from the aircraft nose to the control points (630 zones), i.e. the number of gust lag states can be reduced considerably when the zones are extended. However, with the inclusion of the modal matrix, c.f. equations 20 and 21, the RFA is only valid for one mass/Mach-number combination and is therefore very costly for loop calculations.

\section{A. Trim Results}

Before the simulations can be started, a trim calculation with horizontal flight trim constraints has to be performed.

The strip theory (STR) is based on the same AIC matrix as the VLM, merely the splining is different. Therefore, when a rigid trim is calculated, the results are identical. The differences of the flexible trim calculations are negligible. The DLM matrix for the steady case $(k=0)$ is equivalent to the VLM matrix. However several approximations and transformations are conducted resulting in slight numerical differences. Table 1 summarizes the trim values for angle of attack and incidence of the HTP of the flexible trimmed aircraft.

Table 1. Trim Results

\begin{tabular}{rrrr} 
& STR & VLM & DLM \\
\hline HTP incidence $\delta_{i H}$ & $-4.1^{\circ}$ & $-4.0^{\circ}$ & $-4.3^{\circ}$ \\
angle of attack $\alpha$ & $10.3^{\circ}$ & $10.4^{\circ}$ & $10.5^{\circ}$
\end{tabular}

The high trim angles of attack can be attributed to the missing initial downwash of the aerodynamic model and the relatively low speed.

\section{B. Discrete Tuned Gust}

The discrete gust is defined in JAR/FAR 25.341 as a gust with the shape 1-cos: 


$$
w_{g}=\frac{1}{2} U_{0}\left(1-\cos \frac{2 \pi x}{2 H}\right)
$$

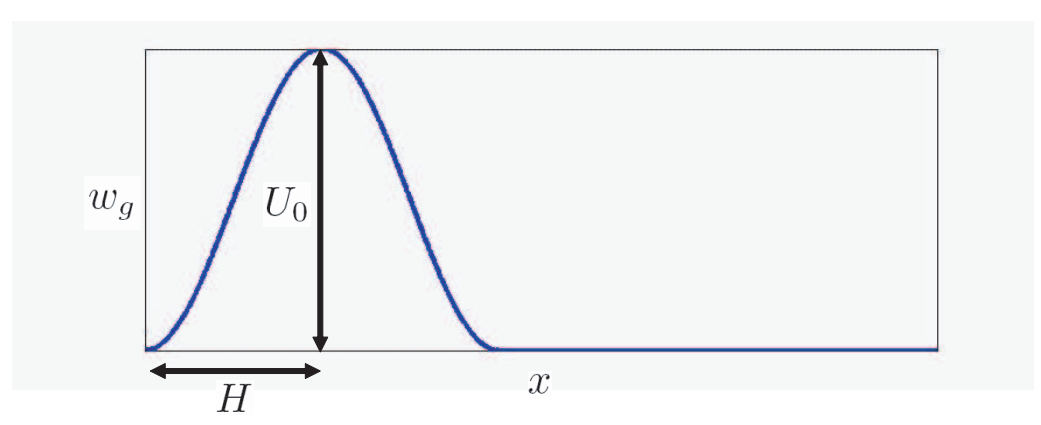

Figure 5. Gust Shape

The gust gradient $H$ is the distance parallel to the airplane's flight path for the gust to reach its peak velocity. For a given gust amplitude several gust gradient distances $H$ between $30 \mathrm{ft}$ and $350 \mathrm{ft}$ have to be calculated to find the critical response for each load quantity. This process is known as gust tuning. The observed quantity is the wing root bending moment $B M_{W i n g}$.

As example four different gust gradients have been calculated with each of the following methods. The steady strip theory (STR), the unsteady strip theory with indicial functions (IFM), the steady Vortex Lattice Method (VLM) and the Rational Function Approximation of the Doublet Lattice Method (DLM).

\section{Results}

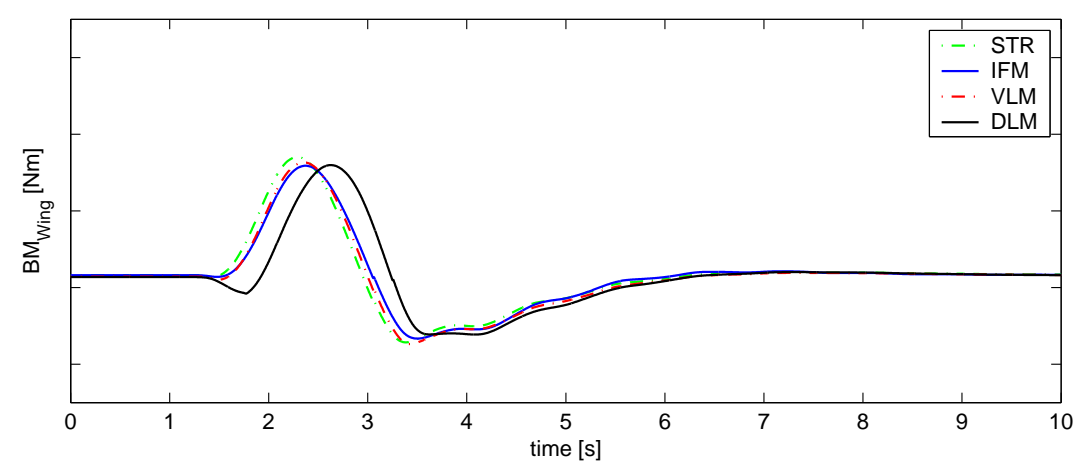

Figure 6. Wing root Bending Moment. Gust Gradient Distance: $335 \mathrm{ft}$

The longest examined gust gradient distance in Figure 6 is closest to a steady case. The differences are expected to be small, the maximum bending moment of all methods lie close together. However there is a considerable phase shift for the DLM. This can be attributed to the lack of modelling of the time lag in downwash between the wing and the empennage. When the wing penetrates the gust, the change of the wing downwash is delayed by the travelling time to the empennage, which is inherently included in the DLM model. Therefore, a downward pitching moment acts before the downwash reaches the empennage, relieving the bending moment in the first moments of the gust penetration. In the steady models however, the change of the wing downwash acts immediately on the empennage. The indicial function method (IFM) is also lacking the the delay in downwash and shows the same behavior as the steady methods.

As the gradient distance is reduced, Figures 7 and 8, the DLM produces considerably higher loads compared the other methods.

When finally considering the shortest gust in Figure 9, the need for gust tuning becomes obvious. The magnitude of the bending moment is reduced in comparison to the previously considered gradient distances. 


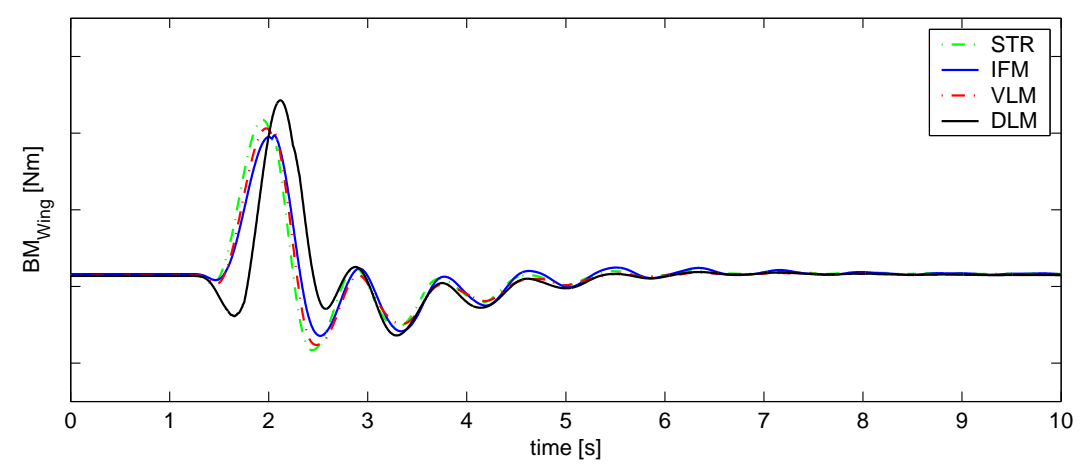

Figure 7. Wing root Bending Moment. Gust Gradient Distance: $164 \mathrm{ft}$

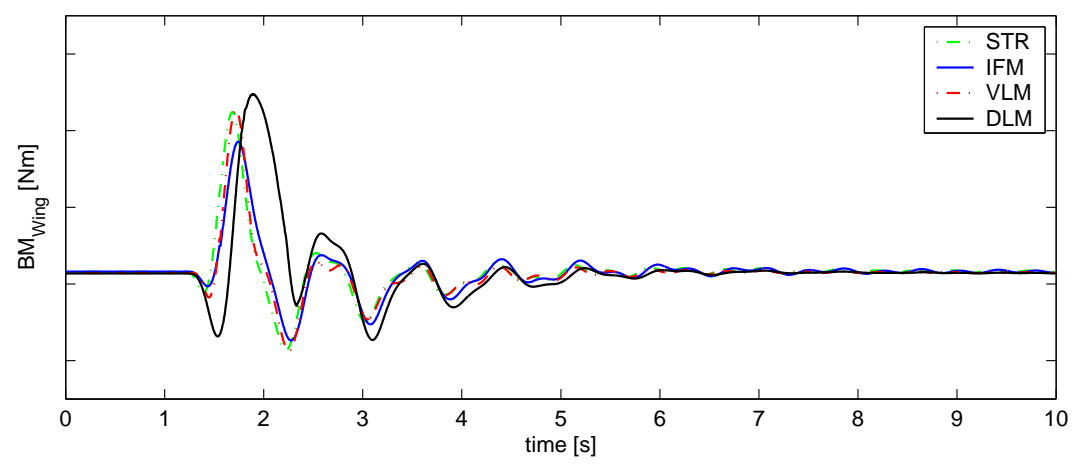

Figure 8. Wing root Bending Moment. Gust Gradient Distance: $84 \mathrm{ft}$

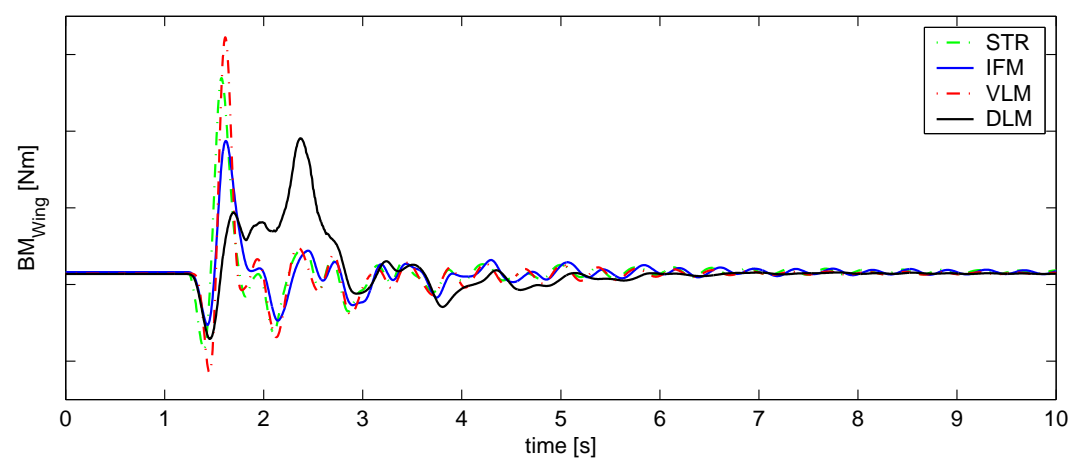

Figure 9. Wing root Bending Moment. Gust Gradient Distance: $42 \mathrm{ft}$

The loads from the steady methods are greatly overestimating the load levels. Interesting to note is also that the maximum load of the DLM is not reached at the first peak, but considerably later, suggesting a high level of aerodynamic interaction.

Table 2 shows the results for the different methods and gradient distances with respect to the maximum load reached with the DLM.

In order to assess the presented methods, it is also important to compare modelling effort and cost of preprocessing and simulation. Table 3 contains an overview of the costs. It has to be mentioned that the simulations were not optimized for speed and a lot of diagnostic output is written, increasing the run times immensely. It also should be noted that the current modelling of DLM constitutes the maximum precision 
Table 2. Discrete Tuned Gust Results

\begin{tabular}{rrrrr} 
& STR & IFM & VLM & DLM \\
\hline $42 \mathrm{ft}$ & 1.039 & 0.889 & 1.136 & 0.896 \\
$84 \mathrm{ft}$ & 0.956 & 0.886 & 0.962 & 1.000 \\
$164 \mathrm{ft}$ & 0.944 & 0.908 & 0.924 & 0.990 \\
$335 \mathrm{ft}$ & 0.858 & 0.838 & 0.845 & 0.839
\end{tabular}

for the gust computation and that the simulation times can be greatly shortened when the number of lag states is reduced.

Table 3. Modelling and Simulation Costs

\begin{tabular}{rrrrr} 
& STR & IFM & VLM & DLM \\
\hline setup of the AIC matrices & $30 \mathrm{~s}$ & $30 \mathrm{~s}$ & $30 \mathrm{~s}$ & $1800 \mathrm{~s}$ \\
unsteady modelling & - & $1 \mathrm{~s}$ & - & $300 \mathrm{~s}$ \\
simulation time for $15 \mathrm{~s}$ & $66 \mathrm{~s}$ & $68 \mathrm{~s}$ & $140 \mathrm{~s}$ & $1800 \mathrm{~s}$ \\
\hline overall & ca. $100 \mathrm{~s}$ & ca. $100 \mathrm{~s}$ & ca. $170 \mathrm{~s}$ & ca. $3900 \mathrm{~s}$
\end{tabular}

\section{Conclusions and Outlook}

The results show the importance of the gust tuning process, since the maximum load can occur at any gust gradient distance. The agreement of the total loads for long gradient distances is quite good, except the fact that the steady methods and the IFM lack the delay in downwash between the wing and the empennage. The implementation of this feature is straight forward and will be done as a next step.

Generally it can be seen that the steady strip theory is always the fastest to react to the gust, because the entire steady state lift acts as soon as the leading edge of the airfoil enters the gust. The VLM reacts slightly slower since the gust reaches each box control point with the respective delayed. The IFM takes into account the build-up of the lift and is the slowest of the three method derived from steady aerodynamics.

The purely steady methods STR and VLM show increasing load levels as the gust gradient distances are reduced. This probably leads to an overestimation of loads. The IFM shows the same sensitivities to the tuning process as the DLM, but underestimates the magnitude of the occurring loads. The inclusion of the effects of the delay in wing downwash remain to be explored. Due to the ease of modelling and fast simulation times, the IFM is the candidate method for predesign studies.

As next steps the lag in downwash will be included in the IFM and for the steady methods STR and VLM as well. Further comparisons with high level methods have to be performed in order to asses the validity of the IFM to estimate gust loads correctly. Gust load computations in the frequency domain can serve as further reference to validate the time domain simulations.

\section{References}

\footnotetext{
${ }^{1}$ Joint Aviation Authorities. Joint Aviation Regulations - 25, Airworthiness Standards: Transport Category Airplanes, volume Subpart C - Structure.

${ }^{2}$ J. Hofstee, T. Kier, C. Cerulli and G. Looye. A variable, fully flexible dynamic response tool for special investigations (VarLoads). In International Forum on Aeroelasticity and Structural Dynamics, 2003.

${ }^{3}$ M.R. Waszak and D.K. Schmidt. Flight dynamics of aeroelastic vehicles. Journal of Aircraft, 25(6):563-571, 1988.

${ }^{4}$ C. S. Buttrill M. R. Waszak and D. K. Schmidt. Modeling and Model Simplification of Aeroelastic Vehicles: An Overview. Technical Report NASA TM-107691, NASA LARC, 1992.

${ }^{5}$ C. Reschke. Flight loads anaysis with inertially couled equations of motion. In AIAA Atmospheric Flight Mechanics Conference. AIAA, 2005.

${ }^{6}$ R. L. Bisplinghoff, H. Ashley, R. L. Halfman. Aeroelasticity. Dover Publications Inc., 1955.

${ }^{7}$ E. Livne and G. Blando. Reduced-order design-oriented stress analysis using combined direct and adjoint solutions. Journal of Aircraft, 38(5):898-909, 2000.
} 
${ }^{8} \mathrm{~J}$. Guyan. Reduction of stiffness and mass matrices. Journal of Aircraft, 3(2):380, 1965.

${ }^{9} \mathrm{~S}$. Hedman. Vortex lattice method for calculation of quasi steady state loadings on thin elastic wings. Technical Report Report 105, Aeronautical Research Institute of Sweden, October 1965.

${ }^{10}$ E. Albano and W.P. Rodden. A doublet lattice method for calculating lifting disturbances of oscillating surfaces in subsonic flows. Journal of Aircraft, 7(2):279-285, 1969.

${ }^{11}$ K.L. Roger. Airplane math modeling methods for active control design. In AGARD Structures and Materials Panel, number CP-228, pages 4-1 - 4-11. AGARD, 1977.

${ }^{12}$ M. Karpel, B. Moulin and P.C. Chen. Dynamic response of aeroservoelastic systems to gust excitation. In International Forum on Aeroelasticity and Structural Dynamics, 2003.

${ }^{13}$ J.G. Leishman. Subsonic unsteady aerodynamics caused by gusts using the indicial method. Journal of Aircraft, 33(5):869-879, 1996

${ }^{14} \mathrm{~T}$. Mauermann. The FEMSPLINE-Method for Connecting Condensed Structural Models and Aerodynamic Models. Technical Report EGLG31 RP0500909, Airbus, Loads and Aeroelastics, 2005.

${ }^{15} \mathrm{G}$. Looye. Integration of rigid and aeroelastic aircraft models using the residualised model method. In International Forum on Aeroelasticity and Structural Dynamics, number IF-046. CEAS/DLR/AIAA, 2005.

${ }^{16}$ J.J. Moré, B.S. Garbow, and K.E. Hillstrom. User guide for MINPACK-1. Technical Report ANL-80-74, Argonne National Laboratory, 1980 